\title{
ALTERNATIVAS DE CONTROLE QUÍMICO PARA CAPIM-AMARGOSO (Digitaria insularis) NA CULTURA DO MILHO
}

\author{
WILTON TAVARES DA SILVA ${ }^{1}$, DÉCIO KARAM ${ }^{2}$, LEANDRO VARGAS ${ }^{3}$ \\ e ALEXANDRE FERREIRA DA SILVA²
}

${ }^{1}$ Graduando Engenharia Agronômica, Universidade Federal de São João-Del-Rei, Estagiário Manejo de Plantas Daninhas Embrapa Milho e Sorgo, Rod MG 424 Km 45, Zona Rural, CEP 35701-970, Sete Lagoas, MG, wilton_tavares@yahoo.com.

${ }^{2}$ Eng. Agr., PhD Plantas Daninhas, Pesquisador Embrapa Milho e Sorgo, Rod MG 424 Km 45, Zona Rural, CEP 35701-970,

Sete Lagoas, MG, decio.karam@embrapa.br.

${ }^{3}$ Eng. Agr., Doutor em Plantas Daninhas, Pesquisador da Embrapa Trigo,

Rodovia BR 285, Km 294, s/n - Zona Rural, CEP 99050-970, Passo Fundo, RS, leandro.vargas@embrapa.br.

${ }^{2}$ Eng.-Agr., D.Sc. em Fitotecnia, Pesquisador em Manejo Integrado de Plantas Daninhas da Embrapa Milho e Sorgo,

Rod MG 424 Km 45, Zona Rural, CEP 35701-970, Sete Lagoas, MG, alexandre.ferreira@embrapa.br

Revista Brasileira de Milho e Sorgo, v.16, n.3, p. 578-586, 2017

\begin{abstract}
RESUMO - O presente estudo teve por objetivo avaliar a eficácia de herbicidas pós-emergentes registrados para a cultura do milho, assim como futuras alternativas de controle químico, no manejo de biótipos de capim-amargoso resistente ao glyphosate. Três experimentos, em condição de casa de vegetação, foram realizados no delineamento de blocos ao caso, com três repetições. Herbicidas de reconhecida eficiência agronômica no controle de gramíneas na cultura do milho (tembotrione, mesotrione e nicosulfuron) e futura alternativas de controle químico (haloxyfop-p-methyl e ammonium-glufosinate) foram aplicados em diferentes períodos de desenvolvimento sobre o capim-amargoso. Foram avaliados os sintomas de intoxicação e a porcentagem de redução do acúmulo da massa de matéria seca (MMS) da infestante após a última avaliação de controle. Os herbicidas nicosulfuron, haloxyfop-p-methyl e ammonium-glufosinate ocasionaram a morte da planta independentemente do momento de aplicação. Tembotrione e mesotrione, apesar de reduzirem a porcentagem de acúmulo da MMS, não ocasionaram morte das plantas. Conclui-se que o nicosulfuron se caracteriza como importante ferramenta no controle de capim-amargoso. As moléculas haloxyfop-p-methyl e ammonium-glufosinate apresentam potencial para serem utilizadas como alternativas de controle químico da infestante. Os herbicidas tembotrione e mesotrione, apesar de reconhecida ação graminicida, não são indicados para o controle do capim-amargoso.
\end{abstract}

Palavras-chave: Zea mays, herbicidas, resistência, planta daninha.

\section{ALTERNATIVES OF CHEMICAL CONTROL FOR SOURGRASS (Digitaria insularis) ON MAIZE CROP}

\begin{abstract}
This study aimed to evaluate the efficacy of registered herbicides for weed control in maize crop and to test future alternatives of chemical control for management of sourgrass biotypes resistant to glyphosate. Three experiments, in the greenhouse, were carried out in a randomized block design with three replicates. Herbicides of recognized agronomic efficiency in grass control on maize (tembotrione, mesotrione and nicossulfuron) and future alternatives of chemical control (haloxyfop-p-methyl e ammonium-glufosinate), as well as, standard herbicide treatment used on maize off-season glyphosate resistant (glyphosate + atrazine), were sprayed on sourgrass plants at different growth periods. The parameters evaluated were intoxication symptoms and the decrease in dry mass accumulation. The herbicides nicossulfuron, haloxyfop-p-methyl and ammonium-glufosinate caused the death of plants, regardless of their developmental stage. In spite of causing a decrease in weed dry mass accumulation, tembotrione and mesotrione did not cause plant death. It is concluded that nicossulfuron is characterized as an important tool in control of sourgrass. The molecules haloxyfop-p-methyl and ammonium-glufosinate have potential to be used as alternatives to control this infestant. The herbicides tembotrione and mesotrione despite recognized grass control were notindicated to control of sourgrass.
\end{abstract}

Keywords: Zea mays, herbicide, resistance, weed. 
A partir da liberação comercial de genótipos resistentes ao glyphosate (GR), a sucessão soja-milho safrinha, realizada em diversas regiões do Brasil, passa a usar este herbicida como principal alternativa química para o controle de plantas daninhas. Contudo, o seu uso continuado tem ocasionado mudanças na comunidade infestante, favorecendo o aparecimento de biótipos resistentes e o aumento de plantas daninhas tolerantes a este herbicida (Barroso et al., 2014). Dentre as plantas daninhas de difícil controle, que têm aumentado de importância no sistema de sucessão soja-milho safrinha, o capim-amargoso (Digitaria insularis) é a espécie que mais merece atenção por parte dos produtores de soja e milho (Licorini et al., 2015). Esta espécie se caracteriza por ser uma planta de difícil controle, em função de sua velocidade de crescimento, da dificuldade de controle de plantas entouceiradas, de sua adaptabilidade a diferentes condições edafoclimáticas, e do aumento da população de biótipos resistentes ao glyphosate no Brasil (Gilo et al., 2016; Carvalho et al., 2011; Mondo et al., 2010; Machado et al., 2006).

O primeiro registro de biótipo de capim-amargoso resistente a glyphosate foi realizado por Adegas \& Gazziero, em 2008, em uma lavoura de soja no município de Guaíra, na região oeste do Paraná (Heap, 2017). Atualmente, outros casos de resistência foram reportados em diversas regiões do Brasil, principalmente, no Estado do Paraná e nas regiões Sudeste, Centro-Oeste e Nordeste do País (Ovejero et al., 2017). Esta espécie apresenta capacidade de germinar em ampla gama de temperatura e luminosidade (Mendonça et al., 2014). Este fato contribui para que o capim-amargoso esteja presente praticamente durante todo o ano nas áreas de produção agrícola. Desta forma, estudos sobre alternativas de controle desta planta daninha são de grande importância para o produtor, pois auxiliam na tomada de decisão para a escolha de uma melhor estratégia para manejo da espécie.

Por meio de resultados de pesquisas, vários trabalhos indicam que, para dessecação de plantas adultas de capim-amargoso em pré-plantio, o glyphosate deve ser associado a herbicidas de ação graminicida que se caracterizam por inibirem a enzima acetil-coenzima A (ACCase), intercalados por uma aplicação de produto de contato ou novamente por outro produto de ação graminicida (Zobiole et al., 2016; Gilo et al., 2016; Correia et al., 2015; Gemelli et al., 2013). Resultados obtidos para a cultura da soja GR indicam a necessidade de aplicações sequenciais de herbicidas de ação graminicida para o controle de plantas de capim-amargoso entouceiradas resistentes ao glyphosate ou uma única aplicação para plantas pequenas (Barroso et al., 2014). O uso excessivo de glyphosate e inibidores da ACCase como única ferramenta no controle químico do capim-amargoso pode ocasionar a seleção de biótipos com resistência múltipla ao glyphosate e a herbicidas deste mecanismo de ação (Ovejero et al., 2017; Gazola et al., 2016). Casos de resistência múltipla de capim-amargoso a glyphosate e a um herbicida inibidor da ACCase (clodinafop) foram recentemente relatados na Bolívia por Franco et al. (2015).

O controle de plantas daninhas no milho safrinha GR consiste, normalmente, na associação de aplicação do glyphosate e atrazine (Bonfleur, 2010). No entanto, na prática, tem-se observado que estes produtos não são efetivos no controle de biótipos de capim -amargoso resistentes ao glyphosate. Estudos abordando o controle de touceiras de amargoso, migrando da soja para milho, demonstram que a utilização de paraquat na pré-colheita da soja seguida pela aplicação de clethodim dois dias após a sua colheita propor- 
ciona melhor flexibilidade para a escolha dos herbicidas utilizados em pós-emergência do milho (Gemelli et al., 2013). No entanto, estratégias de controle de capim-amargoso emergidas durante o desenvolvimento da cultura do milho são escassas na literatura.

Dentre os herbicidas registrados no Ministério da Agricultura, Pecuária e Abastecimento (Mapa), nicosulfuron, tembotrione e mesotrione se destacam como importantes moléculas para o controle de gramíneas na cultura do milho (Rodrigues \& Almeida, 2011). No entanto, nenhuma dessas moléculas é registrada para o controle do capim-amargoso, somente para Digitaria horizontalis, popularmente conhecida como capim-colchão (Agrofit, 2003). No milho GR, o glyphosate se destaca por sua eficiente ação graminicida. Entretanto, tem sido observada ineficácia no controle de plantas adultas e de biótipos resistentes de capim-amargoso (Correia \& Durigan, 2009). A entrada de cultivares de milho tolerante a haloxyfop-pmethyl e ammonium-glufosinate no mercado poderá auxiliar no manejo desta infestante, tendo em vista a eficiência já relatada destes produtos em outras culturas e/ou na dessecação pré-plantio (Gilo et al., 2016; Gemelli et al., 2013). Desta forma, torna-se necessário ampliar o conhecimento sobre os herbicidas disponíveis para serem utilizados na cultura, assim como sobre novas alternativas de controle químico que poderão ser implementadas em função da inserção de genes de tolerância a herbicidas no milho.

O objetivo deste trabalho foi avaliar, em diferentes estádios de aplicação, a eficácia de herbicidas registrados para a cultura do milho, além de futuras alternativas de controle químico para auxiliar no manejo de biótipos de Digitaria insularis resistente ao glyphosate.

Três experimentos foram realizados em 2016, em condições de casa de vegetação, na área experi- mental da Embrapa Milho e Sorgo. O delineamento experimental utilizado nos ensaios foi de blocos ao acaso, com três repetições. Sementes de biótipo de Digitaria insularis resistente ao glyphosate foram coletadas no município de Vicentinópolis-GO, e semeadas em vasos com capacidade para $5 \mathrm{~L}$.

Os tratamentos do Experimento 1 consistiram na aplicação de cinco herbicidas em duas doses, que correspondem à registrada e à metade da dose para $\mathrm{o}$ controle de espécie do gênero Digitaria, além de dois controles, sendo um em que foi realizada a aplicação de atrazine + glyphosate e uma testemunha sem aplicação de herbicida. Os herbicidas utilizados e suas respectivas doses foram haloxyfop-methyl a 31,175 e $62,35 \mathrm{~g} \mathrm{ha}^{-1}$, nicossulfuron a 562,5 e $1.125 \mathrm{~g} \mathrm{ha}^{-1}$, mesotrione a 96 e $192 \mathrm{~g} \mathrm{ha}^{-1}$, tembotrione a 50,4 e 100,8 $\mathrm{g} \mathrm{ha}^{-1}$, ammonium-glufosinate a 350 e $450 \mathrm{~g} \mathrm{ha}^{-1}$ eatrazine + glyphosate na dose de $1.760+1.440 \mathrm{~g} \mathrm{ha}^{-1}$. Os herbicidas foram aplicados no capim-amargoso quando as plantas apresentavam entre 3 e 4 folhas, aos 25 dias após a emergência das plantas.

No Experimento 2, os herbicidas foram aplicados sequencialmente após o tratamento padrão de atrazine + glyphosate. A aplicação da mistura herbicida foi realizada aos 25 dias após a emergência (DAE), quando as plantas apresentavam entre $3 \mathrm{e}$ 4 folhas, e a sequencial realizada 15 dias após (40 DAE). Os tratamentos consistiram na aplicação de quatro herbicidas nas doses registradas para o controle de espécie do gênero Digitaria. Os tratamentos herbicidas corresponderam a atrazine + glyphosate na dose de $1.760+1.440 \mathrm{~g} \mathrm{ha}^{-1}$, haloxyfop-methyl a $62,35 \mathrm{~g} \mathrm{ha}^{-1}$, nicossulfuron a $1.125 \mathrm{~g} \mathrm{ha}^{-1}$, mesotrione a $192 \mathrm{~g} \mathrm{ha}^{-1}$, e tembotrione $100,8 \mathrm{~g} \mathrm{ha}^{-1}$, além de uma testemunha, sem aplicação de herbicida, e um controle somente com a aplicação de atrazine + glyphosate. 
Os tratamentos do Experimento 3 consistiram na aplicação de cinco herbicidas em duas doses, que corresponderam à registrada e à metade dela para o controle de espécie do gênero Digitaria, além de dois controles, sendo um em que foi realizado a aplicação de atrazine + glyphosate e uma testemunha sem aplicação de herbicida. Os produtos utilizados e suas respectivas doses correspondem a haloxyfop-methyl a 31,175 e 62,35 g $\mathrm{ha}^{-1}$, nicossulfuron a 562,5 e $1.125 \mathrm{~g} \mathrm{ha}^{-1}$, mesotrione a 96 e $192 \mathrm{~g} \mathrm{ha}^{-1}$, tembotrione a 50,4 e 100,8 $\mathrm{g} \mathrm{ha}^{-1}$, ammonium-glufosinate a 350 e $450 \mathrm{~g} \mathrm{ha}^{-1}$ eatrazine + glyphosate na dose de $1.760+1.440 \mathrm{~g} \mathrm{ha}^{-1}$. Os herbicidas foram aplicados aos 40 DAE do capim-amargoso quando as plantas se encontravam com 3 a 4 afilhos.

Adjuvantes foram adicionados de acordo com a recomendação de cada produto. Para os herbicidas haloxyfop-methyl e mesotrione foi utilizado óleo mineral a $0,5 \%$ do volume de calda, enquanto que para o nicosulfuron utilizou-se a concentração de $0,1 \%$. Para tembotrione e ammonium-glufosinate foi utilizado óleo vegetal a $0,5 \%$ do volume. A aplicação dos tratamentos com herbicidas foi realizada com um pulverizador costal de pressão constante à base de $\mathrm{CO}_{2}$, equipado com barra de ponta única tipo leque SF 110 02 , sob pressão de $300 \mathrm{kPa}$, que proporcionou volume de calda de $120 \mathrm{~L} \mathrm{ha}^{-1}$.

As características avaliadas nos experimentos foram os sintomas de intoxicação das plantas de capim-amargoso e o acúmulo da massa da matéria seca ao final do período de avaliação. A avaliação de intoxicação das plantas foi realizada com base na escala visual de notas, variando de 0 a $100 \%$, em que 0 significa ausência de sintomas de intoxicação e 100, a morte das plantas. Os sintomas de intoxicação nos Experimentos 1 e 2 foram avaliados aos 14, 28 e 42 dias após a aplicação (DAA). No Experimento 2, os sintomas começaram a ser avaliados a partir da pri- meira aplicação, desta forma, as avaliações realizadas aos 28 e 42 DAA corresponderam, respectivamente, a 14 e 28 dias após a segunda aplicação. No Experimento 3, os sintomas de intoxicação foram avaliados aos 7, 14 e 28 DAA. Ao final da avaliação visual, as plantas foram cortadas rente ao solo e acondicionadas em sacos de papel para aferição da massa da matéria seca. As amostras foram levadas para estufa de circulação de ar forçada a $60{ }^{\circ} \mathrm{C}$, por 72 horas. Após a obtenção da massa de matéria seca das infestantes, os dados foram transformados em porcentagem em relação a testemunha.

As médias dos dados de controle e de massa da matéria seca foram submetidas à análise de variância (ANOVA) pelo teste $\mathrm{F}$ e, quando significativas, comparadas pelo teste de Scott-Knott a 5\% de significância.

Os resultados do presente estudo demonstram que a aplicação única de ammonium-glufosinate e nicosulfuron realizada no capim-amargoso aos 25 DAE, quando as plantas se encontravam com 3 a 4 folhas, ocasionou morte das plantas aos 42 DAA, independentemente das doses utilizadas (Tabela 1). No entanto, o haloxyfop-p-methyl demonstrou ser efetivo somente quando utilizado na dose de $60 \mathrm{~g} \mathrm{ha}^{-1}$. A efetividade dessas moléculas herbicidas no controle do capim-amargoso tem sido comprovada em diversos trabalhos. Timossi (2009) observou que a aplicação de nicosulfuron em rebrotes de capim-amargoso no milho safrinha proporcionou nível de controle de $80 \%$ aos 28 DAA. Gemelli et al. (2013) verificaram que áreas infestadas por rebrotes de capim-amargoso, quando submetidas à aplicação de ammonium-glufosinate, apresentaram redução de aproximadamente $88 \%$ no acúmulo de massa seca da infestante no momento da colheita do milho. Gilo et al. (2016) observaram que haloxyfop-p-methyl ocasionou a morte de 
pantas jovens de capim-amargoso, até quatro perfilhos, aos 28 DAA e reduziu a massa de matéria seca de plantas adultas, em pleno florescimento, em aproximadamente $95 \%$ aos 35 DAA.

No presente estudo, os herbicidas tembotrione e mesotrione, registrados para o controle de $D$. horizontalis (Agrofit, 2003), não foram eficientes, apresentando níveis de controle abaixo de $88 \%$ para o capim-amargoso (Tabela 1). No entanto, a utilização destes produtos ocasionou severa redução no acúmulo da massa da matéria seca da infestante. Estes herbicidas, quando utilizados nas doses de 100,8 $\mathrm{g} \mathrm{ha}^{-1}$ e $192 \mathrm{~g} \mathrm{ha}^{-1}$, reduziram o valor desta variável em, respectivamente, 67 e 79,3\% em relação à testemunha. As doses de 50,4 $\mathrm{g} \mathrm{ha}^{-1}$ e $96 \mathrm{~g} \mathrm{ha}^{-1}$, respectivamente, proporcionaram, aproximadamente, 64\% de supressão do crescimento da infestante. Resultado semelhante foi observado na associação de atrazine + glyphosate, em que a infestante apresentou 39\% da massa da matéria seca em relação à testemunha. A redução na taxa de crescimento da infestante pode favorecer a sua supressão pela cultura (Oliveira Neto,
2011). Este fato pode contribuir para a redução do nível de interferência ocasionado pelo capim-amargoso no rendimento final da cultura. No entanto, a ausência de controle pode favorecer o aumento do banco de sementes, com consequente aumento na infestação de plantas daninhas.

O experimento com aplicação sequencial após o tratamento padrão de atrazine + glyphosate realizada aos $25 \mathrm{DAE}$, quando as plantas apresentavam entre 3 e 4 folhas, e a sequencial realizada 15 dias após (40 DAE), representa uma situação possível de ocorrer no campo. O produtor, após realizar a aplicação de glyphosate + atrazine na pós-emergência do milho GR, percebe a falha de controle no capim-amargoso, necessitando realizar uma aplicação sequencial para promover o controle da infestante. Conforme o esperado, a associação de glyphosate + atrazine não causou a morte da infestante em razão da resistência do biótipo ao glyphosate e da ineficiência do atrazine no controle de gramíneas em estádios avançados de crescimento (Martins et al., 2007). Esta associação de herbicidas, comumente utilizada no milho GR, tem

Tabela 1. Avaliações de controle e redução de massa da matéria seca (MMS) realizadas após a aplicação dos tratamentos, em pós-emergência inicial, estádio de 3 a 4 folhas, das plantas de capim-amargoso.

\begin{tabular}{llllll}
\hline Herbicida & Dose & \multicolumn{2}{l}{ Fitotoxidez $\left.^{\prime} \%\right)$} & \multicolumn{2}{l}{ Redução } \\
& $\left(\mathrm{g} \mathrm{ha}^{-1}\right.$ i.a. $)$ & $14 \mathrm{DAA}^{\prime 1}$ & 28DAA & 42DAA & MMS (\%) \\
\hline \multirow{2}{*}{ Nicosulfuron } & 562,5 & $53,3 \mathrm{e}$ & $92,7 \mathrm{e}$ & $99,3 \mathrm{~d}$ & $100,0 \mathrm{a}$ \\
& 1.125 & $81,7 \mathrm{~g}$ & $100,0 \mathrm{e}$ & $100,0 \mathrm{c}$ & $100,0 \mathrm{a}$ \\
Mesotrione & 96 & $56,7 \mathrm{e}$ & $73,3 \mathrm{c}$ & $76,6 \mathrm{c}$ & $64,3 \mathrm{~d}$ \\
& 192 & $70,0 \mathrm{f}$ & $81,7 \mathrm{c}$ & $88,3 \mathrm{c}$ & $79,3 \mathrm{~b}$ \\
Tembotrione & 50,4 & $33,3 \mathrm{~d}$ & $75,0 \mathrm{c}$ & $78,3 \mathrm{c}$ & $64,0 \mathrm{~d}$ \\
& 100,8 & $33,3 \mathrm{~d}$ & $80,0 \mathrm{~d}$ & $83,3 \mathrm{c}$ & $67,0 \mathrm{c}$ \\
Haloxyfop-p-methyl & 31,175 & $23,3 \mathrm{c}$ & $73,3 \mathrm{c}$ & $80,0 \mathrm{c}$ & $64,0 \mathrm{~d}$ \\
& 62,35 & $98,3 \mathrm{~h}$ & $100,0 \mathrm{e}$ & $100,0 \mathrm{~d}$ & $100,0 \mathrm{a}$ \\
Ammonium-glufosinate & 300 & $95,3 \mathrm{~h}$ & $100,0 \mathrm{e}$ & $100,0 \mathrm{~d}$ & $100,0 \mathrm{a}$ \\
& 450 & $100,0 \mathrm{~h}$ & $100,0 \mathrm{e}$ & $100,0 \mathrm{~d}$ & $100,0 \mathrm{a}$ \\
Atrazine + glyphosate & $1.760,0+1.584,0$ & $8,0 \mathrm{~b}$ & $25,0 \mathrm{~b}$ & $40,0 \mathrm{~b}$ & $61,0 \mathrm{e}$ \\
Testemunha & 0 & $0,0 \mathrm{a}$ & $0,0 \mathrm{a}$ & $0,0 \mathrm{a}$ & $0,0 \mathrm{f}$ \\
\hline CV\% & & 7,59 & 5,39 & 6,7 & 4,37 \\
\hline
\end{tabular}

*Médias seguidas da mesma letra na coluna não diferem entre si pelo teste de Scott-Knott $(\mathrm{p} \leq 0,05)$. - DAA ${ }^{11}=\mathrm{Dias}^{\mathrm{A}} \mathrm{Após}$ Aplicação. 
como objetivo principal o controle de plantas voluntárias de soja e aumento do espectro de ação, principalmente de folhas largas tolerantes ao glyphosate, como corda-de-viola (Ipomoea spp.), trapoeraba (Commelina spp.), erva-de-touro (Tridax procumbens), entre outras espécies. Diante deste cenário, o produtor de milho deve buscar alternativas de controle químico que sejam eficientes no controle do capim-amargoso.

Os herbicidas nicossulfuron e haloxyfop-pmethyl demonstraram ser eficientes no controle do capim-amargoso quando aplicados 15 dias após o uso de atrazine + glyphosate (Tabela 2). Este resultado demonstra o uso potencial de nicosulfuron, herbicida inibidor da enzima acetolactato sintase (ALS), como importante alternativa de controle do capim-amargoso. No entanto, é importante salientar que os genótipos de milho podem apresentar tolerância diferenciada a esta molécula, a depender do seu estádio de desenvolvimento e do intervalo da adubação nitrogenada (López-Ovejero et al., 2003; Guerra et al., 2010). O herbicida haloxyfop-p-methyl se destaca como uma alternativa de uso futuro, tendo em vista a aprovação da liberação comercial de materiais tolerantes a esta molécula herbicida (Comissão Técnica Nacional de Biotecnologia, 2017). No entanto, é importante salientar que, por causa do uso inadequado de her- bicidas deste mecanismo de ação, biótipos com resistência simples a haloxyfop-p-methyl e fenoxaprop-p -ethyl foram identificados na região Centro-Oeste do Brasil no ano de 2016 (Heap, 2017). O uso inadequado de herbicidas deste mecanismo de ação associado ao glyphosate vem despertando a suspeita da presença biótipos com resistência múltipla no País. Licorini (2014), em estudo com biótipos de capim-amargoso coletados no Estado do Paraná, observou que alguns biótipos resistentes a glyphosate não foram controlados pela dose recomendada de clethodim, indicando a possibilidade de resistência múltipla.

Tembotrione e mesotrione, mesmo sendo aplicados 15 dias após a associação de atrazine + glyphosate, demonstraram ser ineficientes no controle do capim-amargoso, porém promoveram a redução de, aproximadamente, 78 e $82 \%$ no acúmulo de massa da matéria seca, respectivamente. Apesar da intoxicação e da redução da massa de matéria seca ocasionadas pela aplicação prévia da mistura herbicida, o capim-amargoso não se tornou mais susceptível a estes herbicidas a ponto de morrer. Gemelli et al. (2013), ao avaliarem diferentes estratégias de controle de rebrotas de capim -amargoso na cultura do milho safrinha, observaram que o tembotrione suprimiu o desenvolvimento da infestante em torno de $90 \%$ ao final do ciclo do milho.

Tabela 2. Avaliações de controle e redução de massa de matéria seca (MMS) referentes a aplicação única e sequencial nas plantas de capim-amargoso, realizadas no estádio de 3 a 4 folhas e 15 dias após.

\begin{tabular}{|c|c|c|c|c|c|c|c|}
\hline \multirow[t]{2}{*}{ Herbicida } & \multirow{2}{*}{ 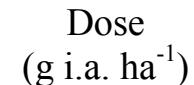 } & Herbicida & Dose & \multicolumn{3}{|c|}{ Fitotoxidez (\%) } & Redução \\
\hline & & Sequencial & ( $\mathrm{g} \mathrm{ha}^{-1}$ i.a.) & $14 \mathrm{DAA}^{/ 1}$ & 28DAA & $42 \mathrm{DAA}$ & $\operatorname{MMS}(\%)$ \\
\hline \multirow{5}{*}{$\begin{array}{l}\text { Atrazine+ } \\
\text { glyphosate }\end{array}$} & \multirow{5}{*}{$\begin{array}{l}1760,0+ \\
1584,0\end{array}$} & - & - & $8,0 \mathrm{~b}$ & $25,0 \mathrm{~b}$ & $39,7 \mathrm{~b}$ & $61,0 \mathrm{~d}$ \\
\hline & & Nicosulfuron & $1.125,0$ & $8,0 \mathrm{~b}$ & $71,66 \mathrm{e}$ & $100,0 \mathrm{e}$ & $100,0 \mathrm{a}$ \\
\hline & & Mesotrione & 192,0 & $7,66 \mathrm{~b}$ & $75,0 \mathrm{f}$ & $81,66 \mathrm{~d}$ & $74,3 \mathrm{c}$ \\
\hline & & Tembotrione & 100,8 & $8,0 \mathrm{~b}$ & $65,0 \mathrm{~d}$ & $77,66 \mathrm{c}$ & $85,3 \mathrm{~b}$ \\
\hline & & Haloxyfop-p- methyl & 62,35 & $8,0 \mathrm{~b}$ & $60,0 \mathrm{c}$ & $100,0 \mathrm{e}$ & $100,0 \mathrm{a}$ \\
\hline Testemunha & - & - & - & $0,0 \mathrm{a}$ & $0,0 \mathrm{a}$ & $0,0 \mathrm{a}$ & $0,0, \mathrm{f}$ \\
\hline $\mathrm{CV} \%$ & - & - & - & 9,43 & 2,38 & 2,38 & 2,62 \\
\hline
\end{tabular}

*Médias seguidas da mesma letra na coluna não diferem entre si pelo teste de Scott-Knott $(\mathrm{p} \leq 0,05)$. - DAA ${ }^{1}=$ Dias Após Aplicação. $^{\circ}$ 
Para o estudo da aplicação dos herbicidas em plantas mais desenvolvidas de capim-amargoso, quando estas apresentavam entre 3 e 4 perfilhos, os herbicidas nicosulfuron, haloxyfop-p-methyl e ammonium-glufosinate se destacaram no controle das plantas, corroborando com os resultados observados nos ensaios anteriores (Tabela 3). Diferentemente do primeiro ensaio, em que a dose mais baixa de haloxyfop-p-methyl não promoveu o controle do capim -amargoso, observou-se neste experimento a morte das plantas mais desenvolvidas. A diferença de comportamento pode ser atribuída, sobretudo, ao aumento da área foliar, o que pode ter favorecido o aumento da interceptação das gotas de herbicidas e, consequentemente, favorecido a ação do produto.

Assim como nos demais experimentos, os herbicidas tembotrione e mesotrione não foram eficientes no controle do capim-amargoso. A dose mais alta de mesotrione resultou em maior supressão de crescimento da infestante, reduzindo o acúmulo de massa da matéria seca em apenas $69 \%$ em relação à testemunha. Apesar de ambas as moléculas herbici- das possuírem boa ação graminicida e serem registradas para o controle de $D$. horizontalis, demonstraram ser ineficientes no controle de D. insularis. Espécies de Digitaria podem apresentar suscetibilidade diferenciada à ação de herbicidas em função de absorção, translocação e metabolismo específico de cada espécie (Tropaldi et al., 2015). Estas características podem justificar a ineficácia destas moléculas no controle do capim-amargoso e, por isto, a importância de novos estudos que possam validar a eficácia de herbicidas no controle desta infestante.

\section{Conclusão}

O herbicida nicosulfuron se destaca como uma alternativa disponível para o controle do capim-amargoso na cultura do milho. Os herbicidas haloxyfop-pmethyl e ammonium-glufosinate apresentam potencial para serem utilizados no controle desta infestante. Os herbicidas tembotrione e mesotrione, apesar de reconhecida ação graminicida, não são indicados para o controle do capim-amargoso.

Tabela 3. Avaliações de controle e redução da massa de matéria seca (MMS) realizadas após a aplicação dos tratamentos, em pós-emergência, estádio de 3 a 4 perfilhos, das plantas de capim-amargoso.

\begin{tabular}{|c|c|c|c|c|c|}
\hline \multirow[t]{2}{*}{ Herbicida } & \multirow{2}{*}{$\begin{array}{l}\text { Dose } \\
\left(\mathrm{g} / \mathrm{ha}^{-1} \text { i. a.) }\right.\end{array}$} & \multicolumn{3}{|c|}{ Fitotoxidez (\% de controle) } & \multirow{2}{*}{$\begin{array}{l}\text { Redução } \\
\operatorname{MMS}(\%)\end{array}$} \\
\hline & & $7 \mathrm{DAA}^{/ 1}$ & 14DAA & 28DAA & \\
\hline \multirow{2}{*}{ Nicosulfuron } & $562,5,0$ & $40,0 \mathrm{c}$ & $51,66 \mathrm{c}$ & $99,3 \mathrm{~d}$ & $100,0 \mathrm{a}$ \\
\hline & 1.125 & $40,0 \mathrm{c}$ & $55,0 \mathrm{~d}$ & $100,0 \mathrm{~d}$ & $100,0 \mathrm{a}$ \\
\hline \multirow{2}{*}{ Mesotrione } & 96 & $30,0 \mathrm{~b}$ & $41,66 \mathrm{~b}$ & $73,33 \mathrm{~b}$ & $47,34 \mathrm{c}$ \\
\hline & 192 & $40,0 \mathrm{c}$ & $50,0 \mathrm{c}$ & $81,66 \mathrm{c}$ & $69,0 \mathrm{~b}$ \\
\hline \multirow{2}{*}{ Tembotrione } & 50,4 & $49,33 \mathrm{~d}$ & $56,66 \mathrm{~d}$ & $71,66 \mathrm{~b}$ & $44,7 \mathrm{c}$ \\
\hline & 100,8 & $41,66 \mathrm{c}$ & $53,33 \mathrm{c}$ & $78,33 \mathrm{c}$ & $46,7 \mathrm{c}$ \\
\hline \multirow{2}{*}{ Haloxyfop-p-methyl } & 31,175 & $45,33 \mathrm{~d}$ & $60,0 \mathrm{e}$ & $100,0 \mathrm{~d}$ & $100,0 \mathrm{a}$ \\
\hline & 62,35 & $48,33 \mathrm{~d}$ & $65,0 \mathrm{f}$ & $100,0 \mathrm{~d}$ & $100,0 \mathrm{a}$ \\
\hline \multirow{2}{*}{ Ammonium-glufosinate } & 300 & $95,0 \mathrm{e}$ & $100,0 \mathrm{~g}$ & $100,0 \mathrm{~d}$ & $100,0 \mathrm{a}$ \\
\hline & 450 & $95,0 \mathrm{e}$ & $100,0 \mathrm{~g}$ & $100,0 \mathrm{~d}$ & $100,0 \mathrm{a}$ \\
\hline Controle & 0 & $0,0 \mathrm{a}$ & $0,0 \mathrm{a}$ & $40,0 \mathrm{~b}$ & $0,0 \mathrm{~d}$ \\
\hline $\mathrm{CV} \%$ & - & 11,17 & 5,39 & 6,7 & 4,37 \\
\hline
\end{tabular}

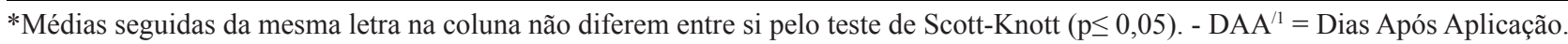




\section{Agradecimentos}

Ao Conselho Nacional de Desenvolvimento Científico e Tecnológico (CNPq) e à Empresa Brasileira de Pesquisa Agropecuária (Embrapa Milho e Sorgo), pela concessão de bolsas. Aos alunos bolsistas e técnicos, pelo auxílio na coleta de dados.

\section{Referências}

AGROFIT. Base de dados de produtos agrotóxicos e fitossanitários: consulta de produtos formulados. Brasília, DF: Ministério da Agricultura, Pecuária e Abastecimento, 2003. Disponível em: <http://extranet.agricultura.gov.br/agrofit cons/principal_agrofit_cons $>$. Acesso em: 9 mar. 2017.

BARROSO, A. A. M.; ALBRECHT, A. J. P.; REIS, F. C.; FILHO, R. V. Interação entre herbicidas inibidores da ACCase e diferentes formulações de glyphosate no controle de capim-amargoso. Planta Daninha, Viçosa, MG, v. 32, n. 3, p. 619-627, 2014.

DOI: $10.1590 / \mathrm{S} 0100-83582014000300018$.

BONFLEUR, E. J. Comportamento da associação entre os herbicidas glifosato e atrazina em um Latossolo vermelho-escuro do bioma cerrado brasileiro. 2010. 84 f. Dissertação (Mestrado em Solos e Nutrição de Plantas) - Escola Superior de Agricultura Luiz de Queiroz, Universidade de São Paulo, Piracicaba, 2010.

DOI: 10.11606/D.11.2010.tde-02082010-170312.

CARVALHO, L. B.; CRUZ-HIPOLITO, H.; GONZÁLEZTORRALVA, F.; COSTA, A.A.; P. L.; CHRISTOFFOLETI, P. J.; PRADO, R. Detection of sourgrass (Digitaria insularis) biotypes resistant to glyphosate in Brazil. Weed Science, Champaign, v. 59, n. 2, p. 171-176, 2011. DOI: 10.1614/WS-D-10-00113.1.

CORREIA, N. M.; DURIGAN, J. C. Manejo químico de plantas adultas de Digitaria insularis com glyphosate isolado e em mistura com chlorimuron-ethyl ou quizalofopp-tefuril em área de plantio direto. Bragantia, Campinas, v. 68, n. 3, p. 689-697, 2009.

DOI: $10.1590 / \mathrm{S} 0006-87052009000300016$.
CORREIA, N. M.; ACRA, L. T.; BALIERO, G. Chemical control of different Digitaria insularis populations and management of a glyphosate-resistant population. Planta Daninha, Viçosa, MG, v. 33, n. 1, p. 93-101, 2015. DOI: $10.1590 / \mathrm{S} 0100-83582015000100011$.

COMISSÃO TÉCNICA NACIONAL DE BIOTECNOLOGIA. Tabela geral de plantas geneticamente modificadas aprovadas comercialmente. Disponível em: $<$ http://ctnbio.mcti.gov.br/liberacao-comercial\#/liberacaocomercial/consultar-processo $>$. Acesso em: 8 mar. 2017.

FRANCO, P.; CONDORI, N.; FLORES H. Resistência múltiple a glifosato y herbicidas inhibidores de ACCasa en Digitaria insularis (L.) Mez ex Ekman y resistencia a glifosato de Amaranthus quitensis H.B.K. en Santa Cruz, Bolivia. In: CONGRESO DE LA ASSOCIACIÓN LATINOAMERICANA DE LAS MALEZAS, 22., 2015, Buenos Aires. Proceedings.... Buenos Aires: Asociación Latino-americana de Malezas, 2015. p. 264.

GAZOLA, T.; BELAPART, D.; CASTRO, E. B.; CIPOLA FILHO, M. L.; DIAS M. F. Características biológicas de Digitaria insularis que conferem sua resistência a herbicidas e opções de manejo. Científica, Jaboticabal, v. 44, n. 4, p. 557-567, 2016.

GEMELLI, A.; OLIVEIRA JÚNIOR, R.; CONSTANTIN, J.; BRAZ, G.; JUMES, T.; GHENO, E.; RIOS, F.; FRANCHINI, L. Estratégias para o controle de capim-amargoso (Digitaria insularis) resistente ao glyphosate na cultura milho safrinha. Revista Brasileira de Herbicidas, Londrina, v. 12, n. 2, p. 162-170, 2013. DOI: 10.7824/rbh.v12i2.201.

GILO, E. G.; MENDONÇA, C. G.; SANTO, T. L. E.; TEODORO, P. E. Alternatives for chemical management of sourgrass. Bioscience Journal, Uberlândia, v. 32, n. 4, p. 881-889, 2016. DOI: 10.14393/BJ-v32n4a2016-32786.

GUERRA, N.; MACIEL, C. D. G.; OLIVEIRA NETO, A. M.; POLETINE, J. P.; RAMOS, G. G. L.; SOLA JÚNIOR, L. C. Seletividade de formulações de nicosulfuron para híbridos de milho em função da época da adubação nitrogenada. Revista Brasileira de Herbicidas, Londrina, v. 9, n. 3, p. 89-99, 2010. DOI: 10.7824/rbh.v9i3.86. 
HEAP, I. International survey of herbicide resistant weeds. Disponível em: <http://www.weedscience.org>. Acesso em: 15 jan. 2017.

LICORINI, L. R. Resistência de biótipos de Digitaria insularis (L.) Fedde aos herbicidas glyphosate e clethodim. 2014. 32 f. Dissertação (Mestrado em Agronomia) - Universidade Estadual do Norte do Paraná, Bandeirantes, 2014.

LICORINI, L. R.; GANDOLFO, M.; SORACE, M.; OSIPE, R.; COSSA, C.; OSIPE, J. Identificação e controle de biótipos resistentes de Digitaria insularis (L.) Fedde ao glyphosate. Revista Brasileira de Herbicidas, Londrina, v. 14, n. 2, p. 148-154, 2015. DOI: 10.7824/rbh.v14i2.394.

LÓPEZ-OVEJERO, R. F.; FANCELLI, A. L.; DOURADONETO, D.; GARCÍA Y GARCÍA, A.; CHRISTOFFOLETI, P. J. Seletividade de herbicidas para a cultura do milho (Zea mays) aplicado em diferentes estádios fenológicos da cultura. Planta Daninha, Viçosa, MG, v. 21, n. 3, p. 413419, 2003. DOI: $10.1590 / \mathrm{S} 0100-83582003000300009$.

MACHADO, A. F. L.; FERREIRA, L. R.; FERREIRA, F. A.; FIALHO, C. M. T.; SANTOS, L. D. T.; MACHADO, M. S. Análise de crescimento de Digitaria insularis (L.) Fedde. Planta Daninha, Viçosa, MG, v. 24, n. 4, p. 641647, 2006. DOI: 10.1590/S0100-83582006000400004.

MARTINS, D.; CARDOSO, L. R.; DOMINGOS, T. V. D.; MARTINS, C. C.; MARCHI, S. R.; COSTA, N. V. Seletividade de herbicidas aplicados em pós-emergência sobre capim-braquiária. Revista Brasileira de Zootecnia, Viçosa, MG, v. 36, n. 6, p. 1969-1974, 2007.

DOI: $10.1590 / \mathrm{S} 1516-35982007000900004$.

MENDONÇA, G. S. D.; MARTINS, C. C.; MARTINS, D.; COSTA, N. V. D. Ecophysiology of seed germination in Digitaria insularis (L.) Fedde). Revista Ciência Agronômica, Fortaleza, v. 45, n. 4, p. 823-832, 2014. DOI: $10.1590 / \mathrm{S} 1806-66902014000400021$.
MONDO, V. H. V.; CARVALHO, S. J. P.; DIAS, A. C. R.; MARCOS FILHO, J. Efeitos da luz e temperatura na germinação de sementes de quatro espécies de plantas daninhas do gênero Digitaria. Revista Brasileira Sementes, Brasília, DF, v. 32, n. 1, p. 131-137, 2010. DOI: 10.1590/S0101-31222010000100015.

OLIVEIRA NETO, A. M. de. Manejo outonal de conyza spp. baseado em glyphosate + 2,4- d, MSMA e amônioglufosinato aplicados isoladamente ou em mistura com herbicidas residuais. 2011. 75 f. Dissertação (Mestrado em Solos e Nutrição de Plantas) - Universidade Estadual de Maringá, Maringá, 2011.

OVEJERO, R. F. L.; TAKANO, H. K.; NICOLAI, M.; FERREIRA, A.; MELO, M. S.; CAVENAGHI, A. L.; OLIVEIRA JR., R. S. Frequency and dispersal of glyphosate-resistant sourgrass (Digitaria insularis) populations across brazilian agricultural production areas. Weed Science, Champaign, v. 65, n. 2, p. 285-294, 2017. RODRIGUES, B. N.; ALMEIDA, F. S. (Ed.). Guia de herbicidas. 6. ed. Londrina: Ed. dos Autores, 2011. 697 p.

TIMOSSI, P. C. Manejo de rebrotes de Digitaria insularis no plantio direto de milho. Planta Daninha, Viçosa, MG, v. 27 , n. 1, p. 175-179, 2009.

DOI: $10.1590 / \mathrm{S} 0100-83582009000100022$.

TROPALDI, L.; VELINI, E. D.; CARBONARI, C. A.; ARALDI, R.; CORNIANI, N.; GIROTTO, M.; FREITAS, I. P. S. Detecção da tolerância de diferentes espécies de capim-colchão a herbicidas inibidores do fotossistema II utilizando a técnica da fluorescência. Ciência Rural, Santa Maria, v. 45, n. 5, p. 767-773, 2015.

DOI: $10.1590 / 0103-8478 \mathrm{cr} 20140506$.

ZOBIOLE, L.; KRENCHINSKI, F.; ALBRECHT, A.; PEREIRA, G.; LUCIO, F. R.; ROSSI, C.; RUBIN, R. Controle de capim-amargoso perenizado em pleno florescimento. Revista Brasileira de Herbicidas, Londrina, v. 15, n. 2, p. 157-164, 2016.

DOI: $10.7824 /$ rbh.v15i2.474. 